\title{
How an Online Health Support Community Responded to the COVID-19 Crisis
}

\author{
Megan Knittel \\ Michigan State University \\ knittel2@msu.edu
}

\author{
Rick Wash \\ Michigan State University \\ wash@msu.edu
}

\begin{abstract}
The scope and severity of the COVID-19 pandemic has introduced new challenges for people seeking health information online. To understand how an existing online community of people living with a chronic health condition navigate meeting their existing health goals alongside the challenges and tensions resulting from COVID-19, we performed a qualitative content analysis of six weeks of discussion in the r/infertility online community. We found that community members collaborated and debated to make dynamic structural and normative changes to their community in accordance with the changing impacts of COVID-19 on their experiences. Additionally, we found that community members information-seeking goals were centered around timelines for their own treatment plans and goals, with the scope of these timelines shifting based on their current state of knowledge of COVID-19. Implications of these findings for supporting health online communities broadly are discussed.
\end{abstract}

\section{Introduction}

The COVID-19 pandemic has major, lasting implications for the lives and well-being of people worldwide. In fighting the pandemic, information-seeking quickly emerged as a challenge for both everyday people seeking accurate, updated information about COVID-19 and for public health officials and governments attempting to communicate information and policy about the pandemic. COVID-19 misinformation is widespread and has manifested in widespread dangerous beliefs that challenge medical standards [1].

Existing work on crisis situations and information-seeking suggests that people experiencing unexpected, disruptive events prioritize certain types of information over others. To this end, people frequently identify a need for information and a lack of quality information sources as a concern [2]. Social media and online communities can support people experiencing the effects of crisis, such as people living in the same place [3] or people with the same pre-existing health condition [4]. But, elements of the COVID-19 situation could challenge these existing trends in crisis communication and collective sense-making online. The unprecedented scale and severity of the COVID-19 pandemic have become compounded with rampant misinformation, political polarization of COVID-19 protective behaviors such as mask-wearing and belief in vaccine efficacy, and the rapidly evolving state of knowledge on COVID-19 best practices. These forces compound to create novel challenges that inhibit finding useful, trustworthy, accurate information [1]. User-driven online communities face challenges with supporting the existing wants and needs of users while also navigating how COVID-19 impacts their communities.

While the informational benefits of health online communities have been well-established [5], this inquiry also expands the potential role of online communities in crisis through a longitudinal approach centered on the role of social ties and self-moderation in how people collect and interpret crisis information while the crisis is developing. Examining a community unified by shared social, personal, and medical experiences offers an opportunity to understand design features and information policy that can support these activities. To this end, we studied a single online community, the Redditr/infertility forum. We examined how an established group of people living with a chronic health condition adapted their existing information practices in their community to the novel challenges posed by COVID-19.

Using the onset of the COVID-19 pandemic and the response on r/infertility is a compelling approach for several reasons. First, infertility is a common experiencee. Over 186 million people worldwide experience infertility, defined as "a disease of the male or female reproductive system defined by the failure to achieve a pregnancy after 12 months 
or more of regular unprotected sexual intercourse" [6]. Additionally, people living with health conditions generally are more likely to use online sources for health information [7]. As a result, using this population as a starting place for understanding how people form and act upon community beliefs surrounding novel health situations could better inform targeted interventions for the general population.

We used qualitative content analysis methodologies to reveal patterns of activity and theorize about how r/infertility evolved as users' needs for the platform changed in response to COVID-19. Over six weeks, we analyzed individuals' discussions to understand the role of this online community at the nexus of chronic health conditions and the onset of COVID-19. Our findings reveal that $r$ /infertility navigated balancing the new constraints of COVID-19 with the core goals and motivations of community members by changing where, when, and how different health topics could be discussed within the community. Additionally, these findings reveal strategies r/infertility participants use to balance informational and other needs with changes to established community rules. These findings provide novel insights into how online communities such as Reddit.com can support positive information-seeking activities across crisis contexts.

\section{Literature Review}

\subsection{Crisis Communication and Information-Seeking}

Seeking information is a major motivator for people navigating crisis [8]. Evidence suggests that crisis information-seeking behavior changes as the crisis itself moves through different stages [9]. Crisis refers to unexpected, disruptive events that challenge the safety and well-being of people [10]. Crisis informatics is an orientation towards understanding crisis events that emphasizes how crisis response is a complex interplay of communication between everyday people, first responders, government agencies, technological tools, and other stakeholders [11].

Information-communication technologies (ICTs) can help people deal with crises [12, 3]. When experiencing crisis, people use ICTs to keep track of new and relevant information [13, 14, 15], find updates about the well-being of their friends and family [16], and create community-driven response initiatives including relief efforts [17]. People turn to online communities and other ICTs when they feel personally impacted by a crisis, are worried about the situation, and distrust [18] or otherwise perceive a lack of information flow
[19] from authorities. HCI work has identified many considerations in designing social media systems for supporting crisis information dissemination, including providing resources for assessing personal risk [12] facilitating trust in authoritative information sources [15], and understanding how to use these platforms for resistance and community action [17].

\subsection{Online Communities and User-Driven Structural and Social Changes}

Many online communities differ from social media platforms like Facebook and Twitter in that they allow users more autonomy over discussion topics and activities. Online communities are often curated around specific experiences and expertise rather than existing social proximity, such as chronic illness online communities [4]. For example, a health online community centered around a rare and poorly understood chronic pain condition called vulvodynia has become a crowd-sourced information repository for people experiencing this condition [4].

However, there is limited work on how community moderators [20] and everyday users [21] may work together [20] (or challenge one another [22]) in intentionally changing the structure of the community itself. In other words, how do participants work together to make community rules, respond to new issues and events, and implement new technical features such as software applications? There is value in understanding how existing health communities collaborate to make structural changes to their community in response to new content and challenges related to the massive disruptions of COVID-19. Examining contextual factors and sense-making practices used by these communities would support emerging calls to incorporate the passion and skills of emergent online communities before and during crisis response [23].

RQ1: Do members of $r$ /infertility alter the structure and content organization practices of their community in reference to the COVID-19 pandemic?

\subsection{Health Online Communities and Managing Disagreement}

Though online communities support health can support information-seeking [24], positive health behavior change [25], disease treatment [26], and other collaborative activities, tensions and disagreement about the state of reality are also common [27] especially in complex and uncertain information spaces such as that surrounding COVID-19 [1]. While organizational contexts have examined strategies for promoting consensus and health disagreement [28], there is limited 
work on how people reach consensus during early stages of crisis communication [8] when uncertainty is high. Thus, online communities are a potentially promising context in which to understand how people manage differences of opinion and other conflicts. Due to their often clearly defined shared community goals [4, 29], the strategies these communities employ to balance the varied desires and perspectives of their members could better inform strategies to overcome information challenges in society at large.

RQ2: How does r/infertility navigate tensions and disagreements surrounding community rules related to COVID-19 related information-sharing and discussion?

\subsection{Navigating Differences in User Goals}

In addition to tensions and disagreements, online communities also face the challenge of managing the wide range of goals participants have for joining the community. User roles in online forums frequently fluctuate based on changes in community goals and resources [21]. To this end, structural role theory posits that online community users vary widely in their motivations to stay engaged to participate in a community [30].

As a result, in the case of a widespread crisis like COVID-19, impacts on user goals in a health online community could be far-reaching and useful predictors of their subsequent behavior in the community. Further, COVID-19 has interrupted access to health care services, among other disruptions [31]. Thus, existing inequities related to health information and medical access may become more manifest in user goals for accessing r/infertility. Examining how community moderators and everyday users balance different and conflicting user goals could inform more effective public health messaging strategies and stronger design approaches for promoting community engagement.

RQ3: What challenges do r/infertility members describe in balancing their COVID-19-related needs alongside their existing goals regarding living with infertility?

\section{Method}

\subsection{Why Reddit?}

We selected Reddit.com for our inquiry for several reasons. First, one of our goals was to capture how online sense-making related to COVID-19 changed over time. Crisis situations evolve quickly and how people respond to them moves in stages [9]. Our focus on change over time allows us to expand on existing work in HCI by capturing how ICT use changes as the crisis is recognized and responded to [32]. To this end, Reddit.com records the date and time comments are posted and organizes content based on age. Additionally, we intentionally selected Reddit.com due to the relative anonymization it offers users. Especially with sensitive topics, such as health, people are more honest under conditions of anonymity [33]. Finally, Reddit.com is a popular news websites and thus has a large amount of discussion for analysis; r/infertility alone has over 13,000 registered participants.

\subsection{Why r/infertility?}

Reddit.com allows users to organize themselves into topic specific sub-communities, create sub-community rules, access the Reddit API to capture comments. r/infertility is a sub-community of Reddit.com. Users can post topics they want to discuss, called threads, that other users can comment on. r/infertility, like each Reddit.com community, hosts a team of moderators who enforce sub-reddit rules and norms, such as through the power to delete comments. For example, a stated and enforced rule on r/infertility is a restriction on mentions of "live children". Most discussion and community activity takes place in threads centered around treatment (such as test results and doctor's visits) and general infertility-related topics. New discussion threads regarding these topics are scheduled to automatically post daily. This entire study was conducted in a single community r/infertility. r/infertility is large and active, thus providing a critical mass for our inquiry. Additionally, Reddit communities vary widely in their norms and practices [34]. Thus, a comparison between different subreddits would make identifying explanatory differences challenging due to broad differences in topic, userbase, and community norms.

We bound the time span of our study from March 2020 to May 2020. This time span was intentionally selected to capture the initial reaction and response to the onset of COVID-19. In addition to coinciding with when COVID-19 began being widely recognized as a global crisis, this time span represented some of the highest levels of uncertainty and information-seeking thus far in the pandemic [35].

\subsection{Data}

After several weeks of preliminary observations of r/infertility, we implemented a keyword 
search to hone our inquiry towards COVID-19 focused discussion. Our search parameters included any thread that included at least one mention of "covid", "covid 19" and/or "covid-19" anywhere in the discussion. This search produced 32 individual threads created between March 10, 2020 and May 21st, 2020. These 32 threads contained 1,935 individual comments. During this time span, 23,341 comments were posted in the community overall. We accessed the Reddit API to scrape copies of the threads on the day they were posted and three days after being posted. We scraped the threads again after 72 hours to allow discussion to naturally progress and taper off. This approach allowed us to verify we captured virtually all discussion in a thread. The researchers did not directly interact with any participants.

\subsection{Analysis}

To analyze the data we used an inductive qualitative content analysis approach [36]. Qualitative content analysis afforded us flexibility and efficacy for capturing theoretical strands in written text [37]. Qualitative content analysis was appropriate to define categories and frequency of specific content in our data [38], which was also our goal. Content analysis also allowed us to capture how details and trends changed over time, in different threads, as formal community rules evolved. Finally, qualitative content analysis allowed us to "systematically describe the meaning" [38] of explicit discussion topics and latent meanings such as context and patterns of discussion.

Our analysis progressed through three primary stages. First, we began by defining our research questions based on our interest in community-driven organization practices, the role of r/infertility norms and enforcement, and how individuals balanced informational needs with other concerns. This first stage of analysis also involved becoming richly engaged with the day to day happenings of the field site, with the first author immersing herself in the community. She followed discussion on r/infertility for several weeks in March 2020 to acquaint herself with the community at large. Based upon this immersion, we selected individual comments as our unit of analysis and used a keyword search to identify relevant discussions.

In stage 2, the first author open-coded the 1,935 comments from the 32 threads. Initial open-coding produced 149 unique codes. We did not use inter-rater reliability as part of our analysis due to our focus on codes as a component of the analytical process rather than an end goal, our interest in dynamic social phenomena, and our inductive approach [39].

After revisiting the data several times and re-evaluating the codes for redundancy and consistency in the data, this codebook was refined to 49 codes. See Table 1 for clusters and examples of specific codes.

Finally, in stage 3, during fall and winter 2020, the first author revisited the codebook to interrogate how well the emerging findings represented the data. To accomplish this, based on Onwuegbuzie and Leech's guide for validity and reliability in qualitative research [40], the first author examined the data and findings for disconfirming evidence and contradictions. These issues were not found, further verifying that the patterns are represented across the data.

In our analysis, we took several steps to protect users' privacy. First, we anonymized usernames and made minor aesthetic changes to the comments to prevent identification. Users are referred to with pseudonyms ('User A', 'User B', etc.) for thus purpose. We lightly edited comments and user quotes to remove potentially identifiable information such as location names or personal medical information. Additionally, at time of this submission, we verified that any comments that had been deleted by users or moderators were removed from our analysis.

\section{Findings}

Our analysis revealed two major findings. First, our data suggest that users' information-seeking needs were driven by time-related information. Participants prioritized information related to time and timelines. Participants repeatedly sought information about how much time, ranging from the specificity of hours to more abstract musings about years in the future, they had to make decisions about their infertility treatment in the face of COVID-19 restrictions. Participants were focused on realigning the idiosyncrasies of their own bodies and current treatment plans with the COVID-19 disruptions in access to medical care and other necessary resources.

Our second major finding is that based on community discussion, changes in community rules and features reflected a negotiation between individuals' evolving personal responses to the crisis and the community's moderation team. Community members adjusted their response not just in terms of the content of the community discussion, but also in terms of how they used the affordances of Reddit.com to support their discussions, including temporarily changing the features of the community itself to support these new goals. This manifested in a specific location in the community for all COVID-19 discussion until participants adapted to having the stress and uncertainty of COVID-19 be a part of their everyday life. 
Table 1. Themes and example codes from our codebook

\begin{tabular}{ll}
\hline \multicolumn{1}{c}{ Coding Themes } & \multicolumn{1}{c}{ Example Codes } \\
\hline $\begin{array}{l}\text { Arguments For or Against Changing Community } \\
\text { Structure }\end{array}$ & $\begin{array}{l}\text { making covid thread; } \\
\text { combining covid treatment threads; } \\
\text { combining covid general threads; } \\
\text { want to avoid covid discussion etc. } \\
\text { anxiety of waiting; } \\
\text { considering swapping clinics to speed up timeline; } \\
\text { issue of age etc. } \\
\text { making the decision to cancel treatment; } \\
\text { anxieties } \\
\text { asking others about current treatment access; } \\
\text { constantly refreshing clinic website etc. } \\
\text { covid and pregnancy uncertainties; } \\
\text { discomfort with eggs in limbo due to covid; } \\
\text { fear of further delays etc. } \\
\text { weelings About COVID-19 Discussion } \\
\text { waiting for new clinic information; } \\
\text { what if delayed over a month; } \\
\text { what if delayed over three month; } \\
\text { what if delayed a year etc. }\end{array}$ \\
\end{tabular}

\subsection{Do members of $r$ /infertility alter the structure and content organization practices of their community in reference to the COVID-19 pandemic?}

We found that r/infertility members prioritized their information-seeking goals around two specific kinds of information. First, participants were especially focused on time and COVID-19: how much time they have to see their doctor, or cancel an appointment, or find a new method of treatment before their hospital shuts down, and so on. As a result, in March and April COVID-19 information-seeking comments almost exclusively focused on figuring out what immediate action should be taken in regard to their individual infertility journeys. User $\mathbf{A}^{\prime} \mathbf{S}$ thread captures many of the recurrent concerns early in the pandemic:

User A: "Has anybody been changing or delaying treatment due to COVID-19? I live in the Boston area ... time is so precious. And my embryos are so precious. Has anyone's doctor mentioned it at all?" (r/infertility)

User $\mathbf{A}^{\prime} \mathbf{s}$ concerns emphasize the role of time. Though time is generally an important and sensitive issue for members of this community [29], the early pandemic response in this community intensified time-based decision-making. People felt pressured to make decisions about their treatment plans quickly due to fears of losing access to medical facilities. Additionally, as User A higlights, much of infertility treatment centers around understanding and responding to the unique biological circumstances of their own body. COVID-19 introduces new challenges with aligning the timeline of one's own body with disrupted treatment access out in the world. As User $\mathbf{A}$ describes, "time is so precious...my embryos are so precious". Likening the concept of time to their own biology emphasizes the intimate connection in this community between COVID-19 disruptions, time factors, and personal treatment goals.

As COVID-19 progressed, from mid-March through the beginning of April, discussion shifted from issues like Is it safe to go to my doctor's appointment tomorrow? or Will my appointment this afternoon be cancelled? towards the uncertainties of the weeks, months, or even years ahead. After it was recognized that the pandemic would cause major disruptions for an uncertain but significant length of time, participants shifted their attention to perceived issues with farther in the future. To this end, User $\mathbf{B}^{\prime} \mathbf{s}$ comment demonstrates a trend in discussion towards more abstract, far future time-based discussions: 
User B: “..WW've been trying for YEARS...now I'm worried that this moratorium is going to last 12 months or more." (r/infertility)

In contrast to early time-based discussions, User B has shifted their attention to farther in the future. Rather than soliciting advice about issues in the near future, as was initially common, discussions became driven by swapping and speculating about what if? scenarios regarding if the pandemic persisted months or years into the future. To this end, replies to User B were centered on individuals' own perspectives on the "moratorium", including how they are adjusting their own plans and offering empathy for User $\mathbf{B}^{\prime} \mathbf{S}$ frustrations. As this comment emphasizes, due to the time-sensitive nature of many individuals' treatment circumstances, even shifts of days or weeks can impact people's perceived ability to pursue treatment at all in the future.

\subsection{How does r/infertility navigate tensions and disagreements surrounding community rules related to COVID-19 related information-sharing and discussion?}

First, our analysis revealed that r/infertility made explicit, formal changes to the community's rules in order to manage the changes in discussion resulting from COVID-19. These changes were temporary shifts in response to community member discussions and feedback. These changes centered around the question How should all of this new discussion about COVID-19 be organized?.

After the first mention of COVID-19 appeared in the community on March 10th 2020, an explosion of activity related to COVID-19 manifested in the community. In addition to allowing users to post their own topics for discussion, r/infertility hosts daily discussion threads related to treatment and other topics. However, after the influx of COVID-19 content, participants repeatedly shared their frustrations about how confusing and stressful it was to have COVID-19 topics discussed randomly in other existing topics. During these early days of the pandemic being recognized worldwide, participants felt that COVID-19 discussions derailed the purpose of existing threads, such as the treatment threads. Others reported the anxiety and stress of COVID-19 not having a designated discussion location impacted their enjoyment of the community as a whole. User E's comment exemplifies these patterns of discussion:

User E: "I find this very difficult too. I've stopped going on the 'treatment' threads in this forum because it's too painful to know that others can keep going while we're completely stuck..." (r/infertility)

As User $\mathbf{E}$ describes, they usually spend time in the treatment threads. But, they describe it as being "too painful" to spend time there in light of the pandemic because of seeing others who can continue treatment "while we're[they're] completely stuck". They describe being stuck due to COVID-19 related pauses in their medical treatment. As User $\mathbf{E}$ goes on to describe, the COVID-19 treatment threads had become a refuge to find others who are "stuck" in their treatment too, but the repeated mentions of COVID-19 in general were painful and inhibited these emotional benefits.

As a result of these discussions, the community moderators created a "superthread" on March 15th in which all discussions related to COVID-19 were directed. Further, they also created a software application so that any use of these terms outside the "superthread" would receive an automatic message reply directing them to the "superthread". They simultaneously modified the written community rules to direct all mentions of COVID-19 and coronavirus to this "superthread":

\section{Moderator Team: "In light of the news and many side discussions concerning anxiety around COVID...Part of what is so valuable about this sub is our imperative to cultivate healthy spaces that don't jeopardize the mental health of our members. ..." (r/infertility)}

As this quote describes, due to the "many side discussions" that sprung up in the first days of COVID-19's impact, and the tensions and emotional upset this appeared to cause many participants, this one "superthread" became the formally approved spot for all COVID-19 discussion. As this quote implies, much of the value r/infertility participants find in using the community is for emotional support and empathy for their unique situations.

Next, throughout March and April 2020, ongoing dialogue between regular users and moderators appeared to play a central role in decisions surrounding adapting community structures to the pressures of COVID-19. A few days after the decision to implement the "superthread", the moderators conducted a community survey about the COVID designated thread. Based on the majority of responses from community members, the moderators agreed to make the COVID-19 discussion thread weekly.

Moderator A: "Wanted to check in with everyone on The Mod Squad's latest post 
on COVID and our strategy for addressing discussion... Our strategy of limiting mentions of COVID in our scheduled threads is not changing, but we wanted to check in on how often (if at all) we repost the COVID superthread... the sub has spoken, weekly covid post coming up." (r/infertility)

As moderator Moderator $\mathbf{A}$ describes here, the "Mod Squad", as the r/infertility moderators are referred to in community discussions, were seeking input about if users wanted to make any adjustments to the frequency of the Covid discussion thread being reposted, "if at all". Based on the majority of responses from community members, the moderators agreed to enact a "weekly covid post".

Finally, starting in late April and early May, the opinion on r/infertility towards keeping COVID-19 discussion in the megathread gradually shifted. Initial adverse emotional reactions to COVID-19 tapered, and in their place rose increasing recognition of needing to adapt all r/infertility discussions with the sustained impacts of COVID-19 in mind, as User F's comment exemplifies:

User F: "At this point all posts are related to COVID. I don't really see the point in separating them. It's kinda weird to see a few people posting about regular cycles still in the daily treatment threads..." (r/infertility)

User $\mathbf{F}^{\prime} \mathbf{S}$ comment from April critiques the COVID-19 threads for leaving a dearth of activity in the "daily treatment threads". They describe the separate COVID-19 thread as unnecessary because, due to the spread of COVID-19 in the United States and its impact on virtually every aspect of everyday life for most people, virtually “all posts [on r/infertility] are related to COVID-19" now.

As a result, the weekly COVID-19 "superthreads" were halted. Based on moderator and user comments, drastically reduced activity levels in the COVID-19 threads as compared to previous weeks, and a growing level of acceptance in the community that COVID-19 was likely going to be around for a while, a member of the moderator team posted this message:

Moderator A: "We are stopping the COVID thread. Please comment in the relevant chat or treatment threads. For all of our sanity, please refrain from posting COVID-related standalone threads for items that really can go in the dailies." (r/infertility)
This shift away from scheduled COVID discussion threads coincided with a shift away from COVID-19 discussion in general. COVID-19 was only mentioned twice in the community through the entirety of May. While it is plausible that the removal of the specific threads indirectly discouraged discussing the pandemic, this shift reflected an on-going, stable pattern of reduced activity in the weekly COVID "superthreads". These shifts in community norms and formal guidelines surrounding COVID-19 discussion reflect a dynamic evolution of r/infertility users' needs and concerns surrounding the pandemic.

\section{Discussion}

As was evidenced in the community, decisions surrounding what topics can be discussed where were made based on back and forth discussions between regular participants and the $r$ / infertility moderator team. Additionally, this community used a strategy of temporarily creating a dedicated space for COVID-19 discussion to help users find information in a consolidated place and support the "mental health" of participants. When critiques in the discussions shifted towards COVID-19 becoming a part of everyday life that people had gotten used to, the rules shifted again with the subsequent elimination of specific COVID-19 threads.

These findings also reveal that members of r/infertility advocated for and benefited from not seeing COVID-19 information in certain places in the online community. Rather than contradicting the fact that many r/infertility members found benefits in sharing what they knew about COVID-19 and their personal experiences with pandemic disruptions, participants simultaneously described feeling overwhelmed, exhausted, and stressed out about constantly seeing COVID-19 material in their community. Creating a separate space for COVID-19 discussion as a strategy to prioritize the "mental health" and "sanity" of community participants during the highly uncertain early days of the pandemic supported participant goals for using the community. When feelings towards COVID-19 shifted and people were more comfortable with discussing it alongside their treatment and other everyday topics, the community decided to change structure yet again to support this.

In understanding the sociotechnical evolution of an established online community facing a novel health crisis, several takeaways emerged. In answering RQ1, our findings revealed that $r$ /infertility did change their community structure based on participants' self-described shifts in goals as a result of COVID-19. 
The uncertainty and emotional distress of COVID-19 described by participants appeared to motivate the community moderators to create a dedicated thread for COVID-19 discussion and implement new software to flag discussions of 'covid' and 'coronavirus' to direct them to this new space. Additionally, our findings demonstrate that these alterations to the structure of the community again changed as time passed and participants' attitudes towards the pandemic shifted. These findings also reflect how information-seeking needs in online communities change in tandem with perceived personal impacts of the crisis situation.

Next, RQ2 was informed by our finding that tensions and disagreements on r/infertility were driven by figuring out how information in the community should be reorganized, if at all, during the influx of COVID-19 discussion. Some participants found including COVID-19 in existing everyday discussions, such as the daily treatment thread, to be stressful and upsetting. Others wanted the information to be more centralized and easy to find. These attitudes shifted over time as well. r/infertility dealt with these tensions by putting power and confidence in the hands of the moderators. The moderators elicited community feedback and attempted to reach solutions informed by majority opinion. Additionally, community members helped each other deal with the emotional upheaval of COVID-19 by sharing their own experiences, successful or otherwise, in continuing to seek treatment despite disruptions.

Finally, in reference to RQ3, we found that tensions and challenges on $r$ /infertility related to the mental health consequences of COVID-19. Additionally, community participants repeatedly described the challenge of (re)planning their treatment plans in the face of the time uncertainties of COVID-19. Time being a major consideration of online communities during crisis is not a novel finding (see: [29]). However, two points from our findings push forward what we know about the time sensitive role of information seeking in crisis. First, rather than predictable possible crisis responses such a focus on personal safety in general or seeking out resources like food and shelter quickly, this community was focused on a different resource: non-life threatening function of their own bodies. Additionally, our findings demonstrate how community discussions on delays changed over time. As the pandemic sprawled from days into weeks and months, information-seeking discussions shifted from hours and days (Will I be able to see my doctor tomorrow?) into abstract aspirations for the future (If COVID-19 is still around next year, will I be able to get treatment for my next cycle?).
These findings suggest several implications and opportunities for future research. First, these data suggest that having fast, flexible options to self-regulate information flows by the ICT users themselves can have benefits for discussants. As r/infertility participants shared, many felt that COVID-19 information was bombarding them across all their online activities and everyday life. As a stated goal of r/infertility is to provide a space to help people experiencing infertility escape some of the pressures of everyday life, too much COVID-19 information is upsetting, overwhelming, and unhelpful. Offering options to curate or 'take breaks' from crisis information sources could actually foster a stronger basis of knowledge and more positive outcomes. Also, incorporating quick and easy to use customization tools to ICT users, particularly those related to curating and organizing information flows based on user feedback, could support more accurate health information and more positive mental health outcomes. Further, our findings suggest that there are considerable benefits to be gained from supporting community members in adjusting these information flows themselves to fit their own needs. Allowing users, including appointed community moderators, to make changes and seek feedback in an iterative cycle helped support the oft-competing informational and emotional needs of community members.

\subsection{Limitations}

A limitation with our approach is that it is challenging to determine to what extent community rules (and, by extension, behavior of moderators in relation to these rules) influence the content of discussions. Moderators have the ability to remove content or move content to other threads in the community, among other things. We do not have a clear picture how much work went into actually enforcing these evolving rules beyond what was visible in the community. In our analysis, we did not come across any comments labelled as deleted by moderators. Additionally, we cannot use this type of trace data to draw conclusions about individual's personal characteristics or actual behavior. Members of this community are simply describing their usage of and reactions to this community; we do not have data about their actual individual use activities. Finally, the people who post on r/infertility are self-selected and likely have attributes relevant to ICT use and health information seeking that differ the average person. Future work would benefit from examining more health contexts to determine what approaches are more or less 
universal.

\section{Conclusion}

To understand how online communities respond over time to crisis events, both in the content of their discussion and formal structures of their communities, we performed a qualitative content analysis of COVID-19 related discussion on $r /$ infertility. We found that the community intentionally changed their technical structures and social rules in accordance with shifting goals and challenges of community participants. Additionally, we found that health information-seeking on r/infertility centered around adjusting timelines due to COVID-19 delays in resource access. These findings suggest that design and practice for promoting accurate, useful information flows in health and crisis online communities generally would benefit from flexible content organization tools, support for keeping track of time-based resources, and tools for supporting mental health and emotional well-being as a core component of effective information-seeking.

\section{Acknowledgements}

This material is based upon work supported by the U.S. National Science Foundation under Grant No. 1350253. We also thank Faye Kollig for her contributions to this draft.

\section{References}

[1] S. Evanega, M. Lynas, J. Adams, and K. Smolenyak, "Coronavirus misinformation: quantifying sources and themes in the COVID-19 'infodemic'," JMIR Preprints, p. 13, 2020.

[2] S. H. Soroya, A. Farooq, K. Mahmood, J. Isoaho, and S. Zara, "From information seeking to information avoidance: Understanding the health information behavior during a global health crisis," Information Processing \& Management, vol. 58, p. 102440, Mar. 2021.

[3] B. Semaan and G. Mark, "'Facebooking' towards crisis recovery and beyond: Disruption as an opportunity," in Proceedings of the ACM 2012 conference on Computer Supported Cooperative Work, (New York, New York, USA), pp. 27-36, 2012

[4] A. L. Young and A. D. Miller, "'this girl is on fire": Sensemaking in an online health community for vulvodynia," in Proceedings of the 2019 CHI Conference on Human Factors in Computing Systems CHI '19, (New York, NY, USA), p. 1-13, Association for Computing Machinery, 2019.

[5] S. Faraj, S. L. Jarvenpaa, and A. Majchrzak, "Knowledge collaboration in online communities," Organization Science, vol. 22, pp. 1224-1239, Feb. 2011. Publisher: INFORMS
[6] "WHO | Infertility definitions and terminology," WHO, 2016. Publisher: World Health Organization.

[7] R. J. Shaw and C. M. Johnson, "Health information seeking and social media use on the internet among people with diabetes," Online Journal of Public Health Informatics, vol. 3, p. ojphi.v3i1.3561, June 2011.

[8] B. Reynolds and M. W. Seeger, "Crisis and emergency risk communication as an integrative model," Journal of Health Communication, vol. 10, pp. 43-55, Feb. 2005. Publisher: Taylor \& Francis _eprint: https://doi.org/10.1080/10810730590904571.

[9] R. Rahmi, H. Joho, and T. Shirai, "An analysis of natural disaster-related information-seeking behavior using temporal stages," Journal of the Association for Information Science and Technology, vol. 70, no. 7, pp. 715-728, 2019. https://asistdl.onlinelibrary.wiley.com/doi/pdf/ 10.1002/asi.24155

[10] R. Soden and L. Palen, "Informating crisis: Expanding critical perspectives in crisis informatics," Proc. ACM Hum.-Comput. Interact., vol. 2, Nov. 2018.

[11] C. Reuter, A. L. Hughes, and M.-A. Kaufhold, "Social media in crisis management: An evaluation and analysis of crisis informatics research," International Journal of Human-Computer Interaction, vol. 34, pp. 280-294, Apr. 2018. Publisher: Taylor \& Francis _eprint: https://doi.org/10.1080/10447318.2018.1427832.

[12] X. Gui, Y. Kou, K. H. Pine, and Y. Chen, "Managing uncertainty: Using social media for risk assessment during a public health crisis," in Proceedings of the 2017 CHI Conference on Human Factors in Computing Systems, CHI '17, (New York, NY, USA), pp. 4520-4533, Association for Computing Machinery, May 2017.

[13] D. Dailey and K. Starbird, “'It's raining dispersants": Collective sensemaking of complex information in crisis contexts," in ACM Conference on Computer Supported Cooperative Work (CSCW 2015), (New York, NY, USA), pp. 155-158, Association for Computing Machinery, Feb. 2015.

[14] L. Palen and S. B. Liu, "Citizen communications in crisis: Anticipating a future of ict-supported public participation," in Proceedings of the SIGCHI Conference on Human Factors in Computing Systems, (New York, NY, USA), p. 727-736, Association for Computing Machinery, 2007.

[15] D. Dailey and K. Starbird, "Social media seamsters: Stitching platforms \&amp; audiences into local crisis infrastructure," in ACM Conference on Computer Supported Cooperative Work (CSCW 2017), (New York, NY, USA), pp. 1277-1289, Association for Computing Machinery, Feb. 2017.

[16] L. Austin, B. F. Liu, and Y. Jin, "How audiences seek out crisis information: Exploring the social-mediated crisis communication model," Journal of Applied Communication Research, vol. 40, pp. 188-207, May 2012. Publisher: Routledge _eprint: https://doi.org/10.1080/00909882.2012.654498.

[17] K. Starbird and L. Palen, "Voluntweeters": Self-Organizing by Digital Volunteers in Times of Crisis, p. 1071-1080. New York, NY, USA: Association for Computing Machinery, 2011

[18] B. Semaan and G. Mark, "Technology-mediated social arrangements to resolve breakdowns in infrastructure 
during ongoing disruption," ACM Transactions on Computer-Human Interaction, vol. 18, pp. 21:1-21:21, Dec. 2011.

[19] Y. L. Huang, K. Starbird, M. Orand, S. A. Stanek, and H. T. Pedersen, "Connected through crisis: Emotional proximity and the spread of misinformation online," in Proceedings of the 18th ACM Conference on Computer Supported Cooperative Work Social Computing, CSCW '15, (New York, NY, USA), p. 969-980, Association for Computing Machinery, 2015.

[20] S. Jhaver, A. Bruckman, and E. Gilbert, "Does transparency in moderation really matter? User behavior after content removal explanations on reddit," Proceedings of the ACM on Human-Computer Interaction: $C S C W$, vol. 3, pp. 1-27, Nov. 2019.

[21] D. Yang, R. E. Kraut, T. Smith, E. Mayfield, and D. Jurafsky, "Seekers, providers, welcomers, and storytellers: Modeling social roles in online health communities," in Proceedings of the 2019 CHI Conference on Human Factors in Computing Systems, pp. 1-14, New York, NY, USA: Association for Computing Machinery, May 2019.

[22] H. Almerekhi, S. b. B. J. Jansen, and c.-s. b. H. Kwak, "Investigating toxicity across multiple reddit communities, users, and moderators," in Companion Proceedings of the Web Conference 2020, WWW'20, (New York, NY, USA), pp. 294-298, Association for Computing Machinery, Apr. 2020.

[23] A. Griswold, "Digital detectives and virtual volunteers: Integrating emergent online communities into disaster response operations," Journal of Business Continuity \& Emergency Planning, vol. 7, pp. 13-25, Jan. 2013.

[24] S. D. Lambert and C. G. Loiselle, "Health information-seeking behavior," Qualitative Health Research, vol. 17, pp. 1006-1019, Oct. 2007. Publisher: SAGE Publications Inc.

[25] H. Taiminen, "How do online communities matter? Comparison between active and non-active participants in an online behavioral weight loss program," Computers in Human Behavior, vol. 63, pp. 787-795, Oct. 2016.

[26] L. d. S. Fernandes, C. Calado, and C. A. S. Araujo, "Social networks and health practices: influence of a diabetes online community on adherence to treatment," Ciência \& Saúde Coletiva, vol. 23, pp. 3357-3368, 2018. Publisher: ABRASCO - Associação Brasileira de Saúde Coletiva.

[27] C. Musco, C. Musco, and C. E. Tsourakakis, "Minimizing polarization and disagreement in social networks," in Proceedings of the 2018 World Wide Web Conference, WWW'18, (Republic and Canton of Geneva, CHE), pp. 369-378, International World Wide Web Conferences Steering Committee, Apr. 2018.

[28] S. Fu, X. Cheng, L. Su, A. Bilgihan, and F. Okumus, "Designing collaboration process facilitation in hotel management teams to improve collaboration performance," International Journal of Hospitality Management, vol. 88, p. 102527, July 2020.

[29] M. Knittel, F. Kollig, A. Mason, and R. Wash, "Anyone else have this experience: Sharing the emotional labor of tracking data about me," Proceedings of the $A C M$ on Human-Computer Interaction: CSCW, vol. 5, pp. 79:1-79:30, Apr. 2021.

[30] E. Akar, S. Mardikyan, and T. Dalgic, "User roles in online communities and their moderating effect on online community usage intention: An integrated approach," International Journal of Human-Computer Interaction, vol. 35, pp. 495-509, Apr. 2019. Publisher: Taylor \& Francis _eprint: https://doi.org/10.1080/10447318.2018.1465325.

[31] R. Blundell, M. C. Dias, R. Joyce, and $\mathrm{X}$. Xu, "COVID-19 and inequalities," Fiscal Studies, vol. 41, no. 2, pp. 291-319, 2020. https://onlinelibrary.wiley.com/doi/pdf/10.1111/ 1475-5890.12232.

[32] W. Norris and S. Voida, "Temporality in crisis informatics: Representations of time in digital humanitarian systems," in Theory Transfers? Social Theory and CSCW Research workshop, held in conjunction with the ACM Conference on Computer-Supported Cooperative Work and Social Computing (CSCW 2017), p. 5, 2017.

[33] M. D. Choudhury and S. De, "Mental health discourse on reddit: Self-disclosure, social support, and anonymity," International Conference on Weblogs and Social Media (ICWSM 2014), vol. 8, May 2014. Number: 1.

[34] B. Dosono and B. Semaan, "Moderation practices as emotional labor in sustaining online communities: The case of aapi identity work on reddit," in Proceedings of the 2019 CHI Conference on Human Factors in Computing Systems, (New York, NY, USA), p. 1-13, Association for Computing Machinery, 2019.

[35] G. Mertens, L. Gerritsen, S. Duijndam, E. Salemink, and I. M. Engelhard, "Fear of the coronavirus (COVID-19): Predictors in an online study conducted in March 2020," Journal of Anxiety Disorders, vol. 74, p. 102258, Aug. 2020.

[36] R. Kozinets, Netnography: The essential guide to qualitative social media research. Thousand Oaks, CA: SAGE Publications Ltd, third edition ed., Nov. 2019.

[37] U. Pfeil and P. Zaphiris, "Applying qualitative content analysis to study online support communities," Universal Access in the Information Society, vol. 9, pp. 1-16, Mar. 2010. Publisher: Springer.

[38] M. Schreier, Qualitative content analysis in practice. Sage publications, 2012.

[39] N. McDonald, S. Schoenebeck, and A. Forte, "Reliability and inter-rater reliability in qualitative research: Norms and guidelines for cscw and hci practice," Proc. ACM Hum.-Comput. Interact., vol. 3, Nov. 2019.

[40] A. J. Onwuegbuzie and N. L. Leech, "Validity and qualitative research: An oxymoron?," Quality and Quantity, vol. 41, pp. 233-249, April 2007. 